\title{
Holding the key to healing
}

Self-healing polymers require dynamic, reversible bonds that can reform on the event of physical or chemical damage. In the case of polymers that rely on non-covalent interactions to heal, these are commonly hydrogen bonds or metal-ligand interactions, and the polymers typically require elaborate chemical and/or physical modifications to achieve self-healing.

Now, writing in Science, Marek Urban and colleagues report a self-healing system that repairs simply using van der Waals interactions within commercially available polymers. Only copolymers with a narrow range of compositions - around a 50:50 molar ratio of the monomers were self-healable, with other

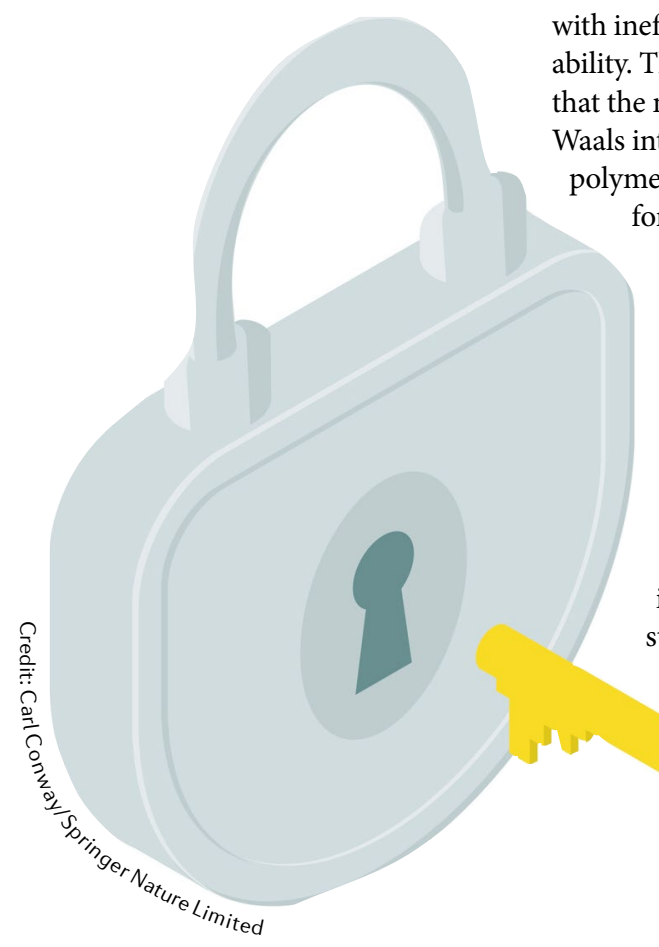

compositions showing either inefficient or no self-repair.

Urban and colleagues prepared copolymers with a range of compositions by varying the ratio of the monomers, methyl methacrylate (MMA) and $n$-butyl acrylate (BA). Also, copolymers (namely, poly(MMA-nBA)) with differing distributions of monomers, either as blocks or as random, alternating patterns, were synthesized. Interestingly, block copolymers with a 50:50 molar ratio did not selfheal, indicating that the alternating arrangement of the slightly longer butyl pendant group with the shorter methyl group is crucial for self-healing to occur.

Molecular dynamics simulations revealed that copolymers with self-healing behaviour had higher cohesive energies than copolymers with inefficient or no self-healing ability. These observations indicate that the non-covalent van der Waals interactions between olymer chains are the driving force for the self-repair of the polymers. "Although van der Waals interactions are rather weak, they are critical in polymeric materials because the interactions span over thousands and millions of polymer repeating units. Thus, collectively these interactions create fairly strong materials, particularly when directional," says Urban.
Copolymers with a MMA/nBA composition of $\sim 50: 50$ and arranged in a preferably alternating manner have strong non-covalent interactions as a result of the interdigitation of the pendant alkyl groups from neighbouring chains. In these copolymers, the chains interact in a key-and-lock-type arrangement; a gap in one chain between two nBA monomer units separated by an MMA monomer unit can host a pendant butyl group from a neighbouring copolymer chain. "If these chains are separated by mechanical force, they will be in thermodynamically unfavourable states, as manifested by increased cohesive energy density values, and tend to return to their initial conformations," explains Urban.

The researchers report a severed 200-micron-thick copolymer film (made from a MMA/nBA monomer ratio of 46:54) that can re-attach in several minutes; however, to regain mechanical properties that are $\sim 90 \%$ of the original material, it takes $\sim 80$ hours. Importantly, if such a material is repeatedly damaged within the same area, this is not detrimental to the self-healing efficiency.

"We have detected similar behaviour in copolymers containing other $n$-alkyl acrylates," explains Urban. "These studies reinforce how significant the design of copolymer chain topologies and the resultant microstructures are on achieving unexpected properties," concludes Urban. "In this case, enabling self-healing to extend the lifespan of commodity polymers." 